\title{
DETERMINAÇÃO VOLTAMÉTRICA DE 4-CLOROFENOL SOBRE O ELETRODO DE DIAMANTE DOPADO COM BORO UTILIZANDO A VOLTAMETRIA DE ONDA QUADRADA
}

\author{
Valber de Albuquerque Pedrosa, Lúcia Codognoto e Luis Alberto Avaca* \\ Instituto de Química de São Carlos, Universidade de São Paulo,CP 780, 13560-970 São Carlos - SP
}

Recebido em 6/1/03; aceito em 6/5/03

\begin{abstract}
ELECTROANALYTICAL DETERMINATION OF 4-CHLOROPHENOL BY SQUARE WAVE VOLTAMMETRY ON BORONDOPED DIAMOND ELECTRODES. The anodic voltammetric behavior of 4-chlorophenol (4-CF) in aqueous solution has been studied on a Boron-doped diamond electrode using square wave voltammetry (SWV). After optimization of the experimental conditions, 4-CF was analyzed in pure and natural waters using a Britton-Robinson buffer with $\mathrm{pH}=6.0$ as the supporting electrolyte. Oxidation occurs at $0.80 \mathrm{~V} v s \mathrm{Ag} / \mathrm{AgCl}$ in a two-electron process controlled by adsorption of the species. The detection limits obtained were $6.4 \mu \mathrm{g} \mathrm{L}^{-1}$ in pure water and $21.5 \mu \mathrm{g} \mathrm{L}^{-1}$ for polluted water taken from a local creek, respectively. The combination of square wave voltammetry and diamond electrodes is an interesting and desirable alternative for analytical determinations.
\end{abstract}

Keywords: 4-chlorophenol; square wave voltammetry; diamond electrodes.

\section{INTRODUÇÃO}

O 4-clorofenol (4-CF) faz parte da família dos clorofenóis, que representa para o ambiente um grave problema de poluição tanto para sistemas aquáticos como terrestres ${ }^{1}$. O 4-CF é um precursor de pesticida e biocida, e também é utilizado na mais diversas atividades industriais incluindo: branqueamento da polpa da celulose na indústria de papel, formação de drogas e está presente em resíduos de gás e óleos industriais ${ }^{2,3}$. O limite permitido em águas naturais é de $100 \mu \mathrm{g} \mathrm{L}^{-1}$, de acordo com "US Environmental Protection Agency"4 (EPA). Outro fator que também despertou interesse para o estudo do 4-CF é por ele ser um típico clorofenol e ter uma estrutura semelhante à de diversos outros pesticidas. Assim, ele pode servir como molécula modelo de uma diversidade de poluentes no desenvolvimento de uma metodologia eletroanalítica.

Os níveis de 4-CF no ambiente são normalmente quantificados usando uma ou mais combinações de técnicas cromatográficas ${ }^{5,6}$. Tais métodos são precisos, sensíveis e seletivos, mas são trabalhosos e relativamente caros. Isto tem despertado o interesse para detecção e quantificação de pesticidas por técnicas eletroanalíticas pois, além de simples e de baixo custo, elas oferecem a possibilidade de se determinar a concentração do poluente diretamente na amostra, sem pré-tratamento ou separação química ${ }^{7}$. Com o desenvolvimento tecnológico as técnicas eletroanalíticas estão sendo cada vez mais utilizadas, destacando-se entre elas, a voltametria de onda quadrada (SWV).

A SWV é uma das técnicas de pulso mais rápida e sensível e os limites de detecção podem ser comparados aos das técnicas cromatográficas e espectroscópicas. Além disso, a análise dos resultados também possibilita a avaliação cinética e mecanística considerando-se espécies adsorvidas ou não à superfície do eletrodo de trabalho. Em função das vantagens apresentadas pela SWV, ela está sendo cada vez mais empregada na determinação de compostos orgânicos ${ }^{8-10}$.

Até o momento, os trabalhos encontrados na literatura com relação ao 4-CF relatam apenas a sua destruição ou degradação por meio de técnicas eletroquímicas e outras (como a degradação microbio-

*e-mail: avaca@iqsc.sc.usp.br lógica ${ }^{11}$ ). Entretanto, não foi localizado nenhum relato sobre a determinação eletroanalítica do 4-CF. Brillas et al. ${ }^{12}$ conseguiram a completa remoção do 4-CF em solução ácida por meio da eletrogeração de $\mathrm{H}_{2} \mathrm{O}_{2}$ a partir de $\mathrm{O}_{2}$ num eletrodo de difusão de gás, na presença de $\mathrm{Fe}^{2+}$ como catalisador. Azzam et al. ${ }^{13}$ usaram eletrodos de $\mathrm{Ti} / \mathrm{TiO}_{2}$ e Ti/RuO para estudar a influência da densidade da corrente, do $\mathrm{pH}$ e da concentração na destruição anódica do 4-CF. Johnson et al. ${ }^{14}$ estudaram a combustão eletroquímica do 4-CF usando um eletrodo de platina revestido com um filme de óxido metálico contendo Ti, $\mathrm{Ru}, \mathrm{Sn}$ e Sb. Finalmente, um trabalho recente do grupo do Prof. Comninellis, na Suíça ${ }^{15}$, descreve a oxidação do 4-CF sobre um eletrodo de diamante dopado com boro em meio ácido, onde, utilizando elevados potenciais anódicos, foi possível conseguir a oxidação completa do referido composto.

Como a maioria dos estudos eletroanalíticos para determinação de pesticidas é realizada sobre o eletrodo de mercúrio, material este extremamente tóxico, a conscientização da alta periculosidade do mesmo leva à busca por novos materiais. Recentemente, um novo material que tem se destacado é o eletrodo de diamante dopado com boro (DDB), em função das suas propriedades atrativas, que incluem uma extensa janela eletroquímica e grande resistência a vários meios agressivos ${ }^{16,17}$. Outro fator importante é a possibilidade de se poder trabalhar com o processo de oxidação do 4-CF sobre o eletrodo de DDB minimizando o efeito de interferentes ${ }^{18}$. Isto porque a maioria dos possíveis metais pesados encontrados em matrizes ambientais é mais facilmente reduzida do que oxidada. Assim, Codognoto et al. ${ }^{19}$ desenvolveram uma metodologia para análise de Pentaclorofenol utilizando o eletrodo de DDB e a SWV e obtiveram um limite de detecção de $5,5 \mu \mathrm{g} \mathrm{L}^{-1}$ para a água pura (Milli-Q) e de $15,5 \mu \mathrm{g} \mathrm{L}^{-1}$ para uma amostra de água poluída de um córrego da região de São Carlos - SP. Já Fushimima et al..$^{20}$ usaram o eletrodo de DDB para detecção eletroquímica do pesticida N-metilcarbamato, e obtiveram um limite de detecção da ordem de $0,6 \mu \mathrm{g} \mathrm{L}^{-1}$.

A combinação da sensibilidade da SWV com as propriedades únicas do eletrodo de DDB é uma excelente alternativa para a determinação de pesticidas. Diante do exposto, o objetivo deste trabalho é o desenvolvimento de uma metodologia para a determinação de 4Clorofenol em águas naturais utilizando a SWV e eletrodo de DDB. 


\section{PARTE EXPERIMENTAL}

\section{Reagentes e soluções}

Foi preparada uma solução estoque do pesticida 4-clorofenol (Aldrich, 99\%) na concentração de $1,5 \times 10^{-3} \mathrm{~mol} \mathrm{~L}^{-1}$ com água ultrapura (Milli-Q). O eletrólito suporte utilizado foi o tampão BrittonRobinson $^{21}$ (BR) $0,1 \mathrm{~mol} \mathrm{~L}^{-1}$. As soluções tiveram o $\mathrm{pH}$ ajustado quando necessário com $\mathrm{NaOH} \mathrm{1,0} \mathrm{mol} \mathrm{L}^{-1}$.

\section{Coleta e preparo das amostras naturais}

As amostras de águas naturais foram coletadas em três pontos diferentes, na cidade de São Carlos: no ponto 1 a amostra foi retirada do poço artesiano do SAAE (serviço de Autônomo de Água e Esgoto, São Carlos) que abastece parte da cidade, no ponto 2 a amostra foi coletada no Córrego do Gregório antes dele cortar a cidade (próximo à nascente) e, finalmente, no ponto 3 a amostra foi retirada do Córrego Monjolinho próximo a residências e criadouros de animais, onde o rio já recebe dejetos domésticos. Para a coleta de águas naturais foram utilizados frascos plásticos, destinados apenas às coletas das mesmas. Estes frascos foram previamente esterilizados para evitar outros tipos de contaminação. Após a coleta, as amostras foram armazenadas no refrigerador na temperatura de aproximadamente $4{ }^{\circ} \mathrm{C}$, por um prazo máximo de cinco dias para a realização dos experimentos.

\section{Medidas eletroquímicas}

As medidas eletroquímicas foram realizadas utilizando diretamente as amostras de águas naturais como solvente, sem nenhum tratamento prévio ou separações. Elas foram somente tamponadas em pH 6,0 com tampão BR 0,1 mol L-1.

As curvas analíticas foram obtidas pelo método de adição de padrão. Para a realização das curvas de recuperação, uma quantidade conhecida de 4-CF foi adicionada ao eletrólito suporte e, a seguir, a concentração de 4-CF adicionada foi determinada pelo método de adição padrão; desta forma obteve-se o valor da recuperação aparen$t^{22}$. As medidas foram feitas em triplicata para cada amostra.

\section{Instrumentação}

A célula eletroquímica utilizada para a determinação eletroanalítica do 4-CF foi uma célula convencional de compartimento único com orifícios para encaixe dos eletrodos. Como eletrodo de trabalho foi utilizado o eletrodo de diamante dopado com boro com área de $0,62 \mathrm{~cm}^{2}$ que foi gentilmente cedido pelo Dr. W. Haenni do Centro Suíço de Eletrônica e Microtécnica (CSEM), Neuchâtel, Suíça. O eletrodo de referência utilizado foi o $\mathrm{Ag} / \mathrm{AgCl}\left(\mathrm{KCl} 3,0 \mathrm{~mol} \mathrm{~L}^{-1}\right)$ e como eletrodo auxiliar foi utilizada uma placa de platina.

Para realização das medidas eletroquímicas utilizou-se o potenciostato/galvanostato 273A da EG\&G PARC, acoplado com o programa M-270 também da PAR.

Para a validação da metodologia foi utilizada a técnica de cromatografia líquida de alta eficiência (HPLC). Utilizando um sistema (HPLC) modelo LC - 10AT Shimadzu com detector de UV-VIS modelo SPD10AVP e coluna LiChrosobr ${ }^{\circledR}$ RP-18 $(5 \mathrm{~mm})$ da Merck, a fase móvel utilizada foi acetonitrila/água (acidificada com $1 \%$ v/v ácido acético) na proporção $60 / 40 \mathrm{v} / \mathrm{v}$, com fluxo de $1,0 \mathrm{~mL}$ por min em condição isocrática, o comprimento de onda monitorado foi de $280 \mathrm{~nm}$.

\section{RESULTADOS E DISCUSSÃO}

O 4-clorofenol em tampão BR 0,1 $\mathrm{mol} \mathrm{L}^{-1}$ e pH = 6,0 apresentou somente um pico de oxidação em torno de $0,80 \mathrm{~V}$. Na Figura 1 está apresentado um voltamograma de onda quadrada para o 4-CF com as separações de corrente. Observa-se que é um voltamograma de onda quadrada de um sistema irreversível ${ }^{23}$.

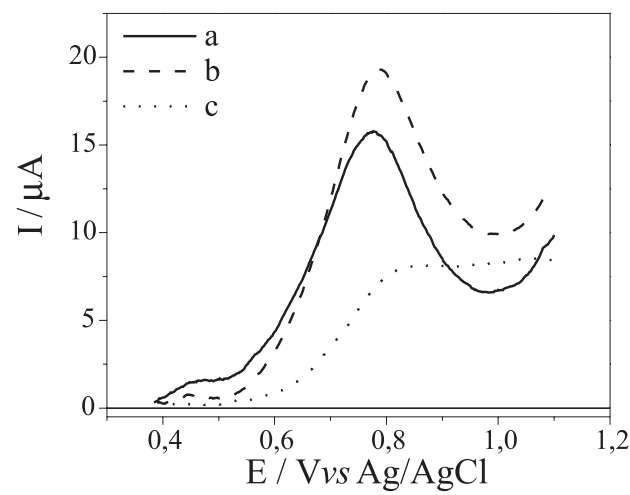

Figura 1. Voltamograma de onda quadrada do 4-CF $\left(1,5 \times 10^{-5} \mathrm{~mol} \mathrm{~L}^{-1}\right.$, tampão BR 0,1 mol $L^{-1} \mathrm{pH}=6,0, \mathrm{f}=100 \mathrm{~s}^{-1}, a=50 \mathrm{mV}, \Delta E_{s}=2 \mathrm{mV}$ ). a) corrente resultante, $b$ ) corrente direta, c) corrente reversa

Um passo importante no desenvolvimento de uma metodologia eletroanalítica é a otimização dos parâmetros que possam influenciar na resposta voltamétrica. Para isto foi realizado um estudo da influência do $\mathrm{pH}$ do meio e dos parâmetros da SWV, como freqüência da onda quadrada $(f)$, amplitude da onda quadrada $(a)$ e incremento de varredura $\left(\Delta E_{s}\right)$, buscando, desta forma, o melhor sinal analítico.

\section{Influência do pH do meio}

O potencial do pico do 4-CF é fortemente influenciado pela variação do $\mathrm{pH}$ do meio. Para valores de $\mathrm{pH}$ menores que 9,0 há um deslocamento de $\mathrm{E}_{\mathrm{p}}$ para valores menos positivos com o aumento do pH. Já para valores de pH acima de 9,0, este parâmetro não influencia na resposta eletroquímica. Na Figura 2 observa-se a variação do $\mathrm{E}_{\mathrm{p}}$ em função do $\mathrm{pH}$, onde foram obtidas duas retas cuja interseção em 9,4 corresponde ao $\mathrm{pK}_{\mathrm{a}}$ do 4-CF, valor este muito próximo ao encontrado na literatura que é de $9,2^{24}$. $\mathrm{O}$ pH de trabalho escolhido foi o de 6,0, pois neste $\mathrm{pH}$ se obtém uma melhor resposta voltamétrica e a corrente de pico apresenta uma maior intensidade de corrente (dados não mostrados). Além disso, a utilização de um meio francamente alcalino poderia danificar o eletrodo de DDB, pois o filme é depositado sobre um substrato de silício.

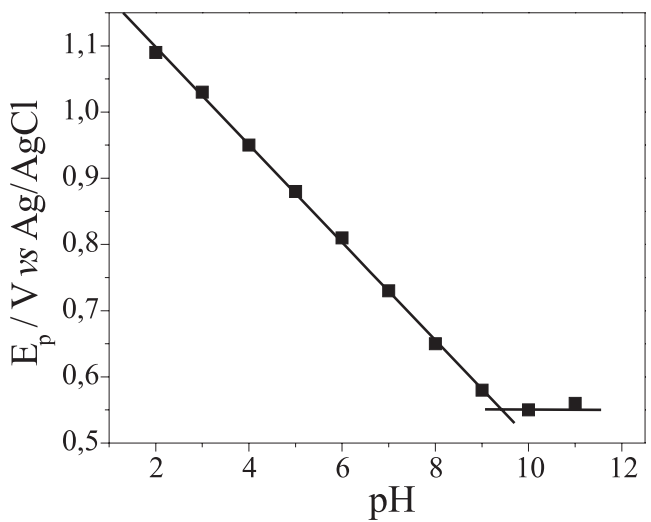

Figura 2. Variação do potencial de pico com o pH para o 4-clorofenol $\left(1,5 \times 10^{-5} \mathrm{~mol} \mathrm{~L}^{-1}\right.$, tampão BR 0,1 $\left.\mathrm{mol} \mathrm{L}^{-1}\right)$ 


\section{Influência da freqüência $(f)$}

A freqüência da onda quadrada é um dos mais importantes parâmetros da SWV, pois é ela que determina a intensidade dos sinais e, conseqüentemente, a sensibilidade do método.

A Figura 3 mostra os voltamogramas de onda quadrada obtidos para o 4-CF em função da variação da freqüência. Observa-se que, com o aumento no valor da freqüência, ocorre um aumento proporcional na intensidade de corrente de pico e também ocorre o deslocamento dos potenciais de pico para regiões mais positivas. No entanto, frequiências acima de $100 \mathrm{~s}^{-1}$ não influenciam de maneira significativa na intensidade do sinal, por este motivo a freqüência de trabalho escolhida foi a de $100 \mathrm{~s}^{-1}$, onde se obtém um voltamograma bem definido, com maior corrente de pico.

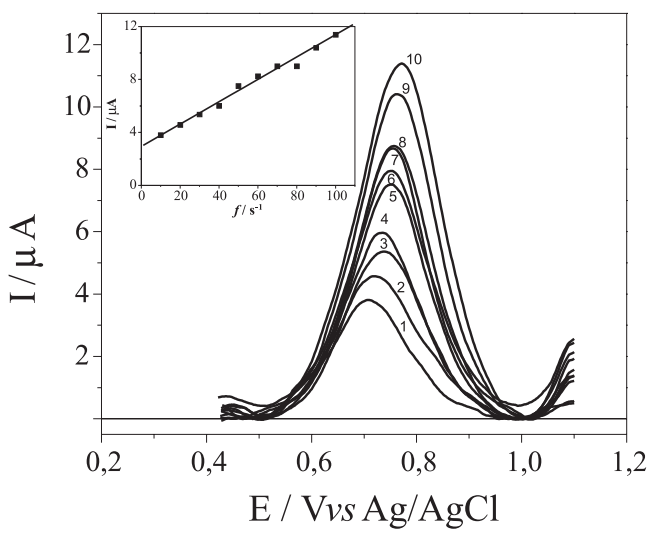

Figura 3. Influência da variação da freqüência da onda quadrada no aspecto geral dos voltamogramas do 4-CF $\left(1,5 \times 10^{-5} \mathrm{~mol} \mathrm{~L}^{-1}, \mathrm{pH}=6,0\right.$ tampão $B R$ 0,1 mol $L^{-1}, a=50 \mathrm{mV}, \Delta E_{s}=2 \mathrm{mV}$ ). Frequiências: 10 (1), 20 (2), 30 (3), 40 (4), 50 (5), 60 (6), 70 (7), 80 (8), 90 (9), $100 \mathrm{~s}^{-1}$ (10). Inserção: Dependência da corrente de pico com a freqüência para o 4-clorofenol

Segundo a teoria proposta por O'Dea et al. ${ }^{25}$ para sistemas totalmente irreversíveis com processos controlados pela adsorção das espécies, a intensidade de corrente varia linearmente com a frequiência de aplicação dos pulsos.

A inserção na Figura 3 mostra o comportamento da corrente de pico em função da variação da freqüência da onda quadrada para o 4-CF. Observa-se que a intensidade de corrente de pico varia linearmente com a frequiência da onda quadrada, mostrando que o processo de oxidação do 4-CF sobre o eletrodo do DDB é irreversível e controlado por adsorção.

De acordo com os critérios de diagnósticos da voltametria de onda quadrada, a dependência dos potenciais de pico com o logaritmo da freqüência para reações totalmente irreversíveis com reagentes e/ou produtos adsorvidos é linear e apresenta uma inclinação de $^{26}$ :

$\frac{\Delta E_{p}}{\Delta \log f}=\frac{2,3 R T}{\alpha n F}$

onde: $\alpha$ é o coeficiente de transferência eletrônica e n o número de elétrons envolvidos na reação eletródica.

Na Figura 4 está apresentado o comportamento do potencial de pico em função do logaritmo da freqüência, para o 4-clorofenol. Observa-se um comportamento linear com inclinação de 0,0635. Aplicando-se a Equação (1) tem-se que $\alpha \mathrm{n}=0,94$, o que corresponderia a um valor de $\alpha$ de 0,47 e dois elétrons por molécula de pesticida envolvidos no processo de oxidação. Estes resultados estão de acordo com o mecanismo proposto na literatura e apresentado no esquema a seguir, onde o produto final é a benzoquinona ${ }^{15}$ :

$$
\mathrm{C}_{6} \mathrm{H}_{5} \mathrm{ClO} \underset{-\stackrel{-\mathrm{H}^{+}}{\longrightarrow}}{\longrightarrow} \mathrm{C}_{6} \mathrm{H}_{4} \mathrm{ClO}^{+} \underset{-\mathrm{HCl}, \mathrm{H}^{+}}{\stackrel{+\mathrm{H}_{2} \mathrm{O}}{\longrightarrow}} \mathrm{C}_{6} \mathrm{H}_{4} \mathrm{O}_{2}
$$

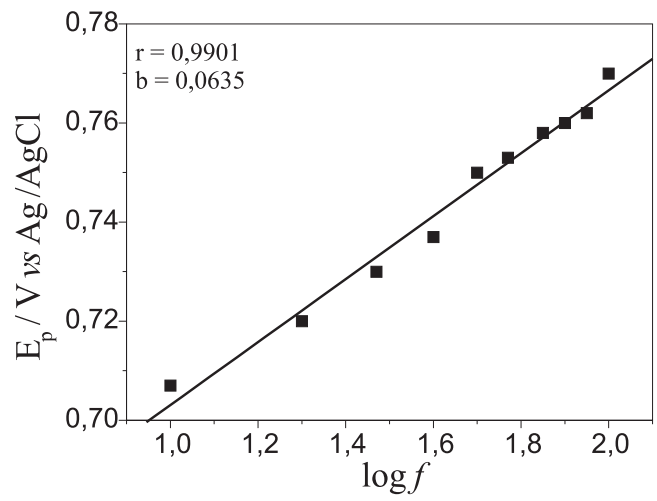

Figura 4. Dependência do potencial de pico com o logaritmo da freqüência da onda quadrada (dados extraídos da Figura 3)

\section{Influência da amplitude (a)}

Para sistemas redox totalmente irreversíveis, a sensibilidade analítica na voltametria de onda quadrada também sofre grande influência com a variação da amplitude da onda, isto porque a largura de meia onda se mantêm constante para amplitudes maiores que $20 \mathrm{mV}^{23}$. A Figura 5 mostra o efeito da variação da amplitude na corrente de pico para o 4-CF. Observa-se que para valores de amplitudes menores que $50 \mathrm{mV}$, o aumento da intensidade de corrente é linear. Adicionalmente, amplitudes maiores parecem não atuar de modo significativo na sensibilidade para propósitos analíticos. Em função disto, optou-se em trabalhar com amplitude da onda quadrada de $50 \mathrm{mV}$.

Considerando-se somente a parte linear do gráfico na Figura 5, é possível calcular a concentração superficial da espécie adsorvida $(\Gamma)$, por meio do coeficiente angular da reta e auxílio da Equação $(2)^{26}$ :

$I_{p}=(5 \pm 1) 10^{2} q \alpha n^{2} F f a \Delta E_{s} \Gamma$

onde q é a área do eletrodo, $\mathrm{n}$ o número de elétrons, $\alpha$ o coeficiente de transferência eletrônica, F a constante de Faraday, $a$ a amplitude da onda quadrada, $\Delta \mathrm{E}_{\mathrm{s}} \mathrm{o}$ incremento de varredura e $f$ a freqüência da onda quadrada.

O valor obtido de concentração superficial $(\Gamma)$ da espécie adsorvida para o 4-CF foi igual a $5,0 \times 10^{-11} \mathrm{~mol} \mathrm{~cm}^{-2}$. Para comparação com este valor, calculou-se a área total de uma molécula de

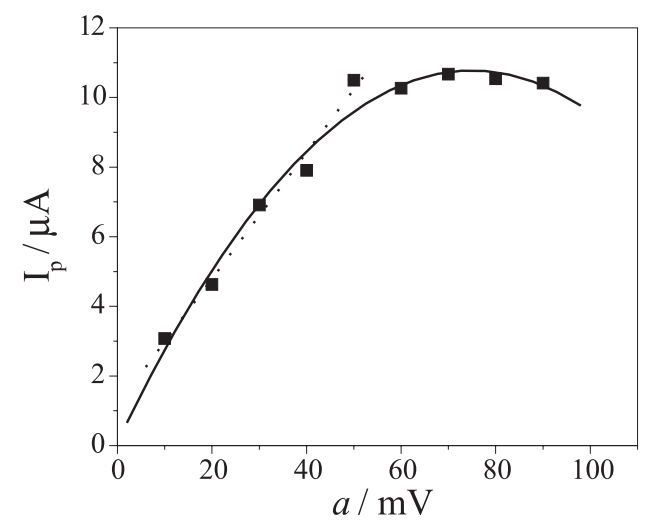

Figura 5. Dependência da corrente de pico com variação de amplitude da onda quadrada para o 4-CF $\left(1,5 \times 10^{-5} \mathrm{~mol} \mathrm{~L}^{-1}\right.$, em tampão BR 0,1 $\mathrm{mol} \mathrm{L}^{-1}$, $\left.p H=6,0, f=100 \mathrm{~s}^{-1}, \Delta E_{s}=2 \mathrm{mV}\right)$ 
4- $\mathrm{CF}^{27}$ que apresentou o valor de $2,5 \times 10^{-14} \mathrm{~cm}^{2}$. Supondo uma cobertura total da superfície eletródica por moléculas de 4-CF, o máximo valor possível seria de $6,5 \times 10^{-11} \mathrm{~mol} \mathrm{~cm}^{-2}$. Devido às aproximações envolvidas nesses cálculos, pode-se dizer que o valor derivado da equação (2) reflete praticamente uma saturação da superfície eletródica com moléculas de 4-CF.

\section{Efeito do incremento de varredura $\left(\Delta \mathrm{E}_{\mathrm{s}}\right)$}

A velocidade efetiva na voltametria de onda quadrada é o resultado do produto da frequiência pelo incremento de varredura. Deste modo, um incremento de varredura maior pode aumentar o sinal obtido e, assim, melhorar a sensibilidade do método ${ }^{23}$. No entanto, com incrementos maiores pode ocorrer um alargamento nos picos obtidos e, assim, a resolução do voltamograma pode ser comprometida. Conseqüentemente, este é um parâmetro que também dever ser analisado.

Na Figura 6 é apresentada a dependência do potencial e da intensidade de corrente de pico em função da variação do incremento de varredura para o 4-CF. Observa-se que, com o aumento do incremento de varredura, ocorre deslocamento do potencial de pico para região mais positiva e que, para incrementos maiores que $2 \mathrm{mV}$, a intensidade de corrente não sofre um aumento muito significativo para fins analíticos, desta forma optou-se em trabalhar com este valor de incremento de varredura $(2 \mathrm{mV})$.

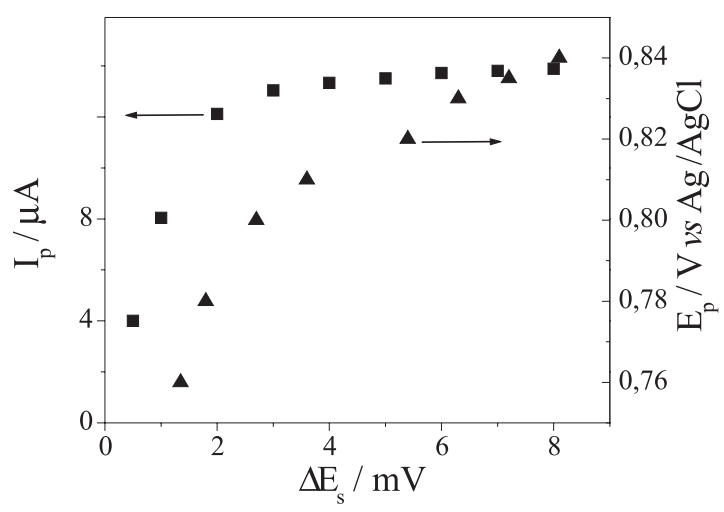

Figura 6. Efeito da variação do incremento de varredura sobre as correntes (ם) e potenciais $(\mathbf{\Delta})$ de pico para o $4-C F 1,5 \times 10^{-5} \mathrm{~mol} \mathrm{~L}^{-1}$, tampão $B R$ $0,1 \mathrm{~mol} \mathrm{~L}^{-1}, \mathrm{pH}=6,0, f=100 \mathrm{~s}^{-1}$ e $a=50 \mathrm{mV}$

\section{Curva analítica para o 4-CF}

Estabelecidas as melhores condições para a determinação do pico de oxidação do 4-CF, a curva analítica em água pura foi construída pelo método da adição de padrão. Os voltamogramas de onda quadrada obtidos para cada concentração de 4-CF são apresentados na Figura 7. Observa-se que a corrente de pico aumenta proporcionalmente com o aumento da concentração e que praticamente não ocorrem deslocamentos nos potenciais de pico. A inserção na Figura 7 corresponde à curva analítica para o 4-CF em água pura, que apresentou linearidade no intervalo de concentração de 0,72 a $3,90 \times 10^{-5} \mathrm{~mol} \mathrm{~L}^{-1}$.

Para a determinação do limite de detecção utilizou-se o desvio padrão da média aritmética de dez voltamogramas de brancos obtida das correntes medidas no mesmo potencial do pico voltamétrico de oxidação do 4-CF, e a relação ${ }^{28}$ :

$L D=\frac{3 S_{B}}{b}$

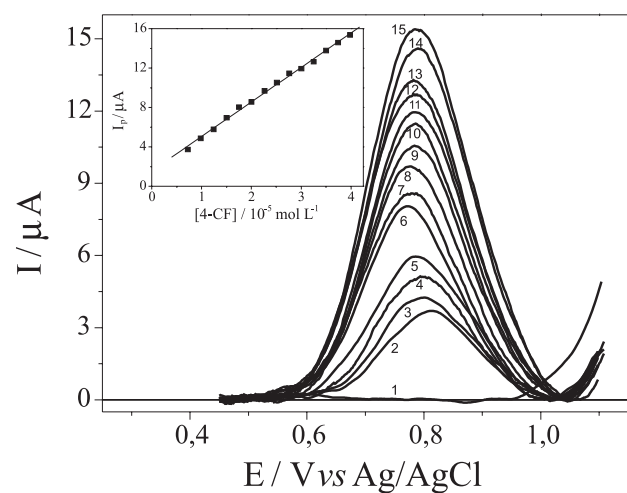

Figura 7. Voltamogramas de onda quadrada para o 4-CF em diferentes concentrações com $f=100 \mathrm{~s}^{-1}, a=50 \mathrm{mV}, \Delta E_{s}=2 \mathrm{mV}$, tampão BR 0,1 $\mathrm{mol}$ $L^{-1}, p H=6.0$. Concentrações: 0 (1) 0,72 (2); 0,98 (3); 1,20 (4); 1,50 (5); 1,70 (6); 2,00 (7); 2,20 (8); 2,50 (9), 2,70 (10), 3,00 (11), 3,20 (12), 3,50

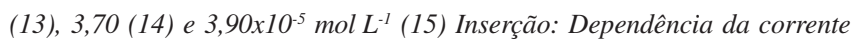
de pico com a concentração para o 4-Clorofenol

onde $\mathrm{S}_{\mathrm{B}}$ é o desvio padrão da média aritmética e b o valor do coeficiente angular da reta de calibração.

O limite de detecção obtido foi de 5,0x $10^{-8} \mathrm{~mol} \mathrm{~L}^{-1}\left(6,4 \mu \mathrm{g} \mathrm{L}^{-1}\right)$. A repetibilidade das medidas foi determinada por medidas sucessivas de cinco soluções diferentes de 4-CF na concentração de $1,5 \mathrm{x}$ $10^{-5} \mathrm{~mol} \mathrm{~L}^{-1}$, com coeficiente de variação de $1,1 \%$ e o desvio padrão relativo (RSD) calculado para o mesmo nível de concentração foi de $1,3 \%(\mathrm{n}=5)$.

Para a validação destes resultados foi obtida uma curva analítica por cromatografia líquida de alta eficiência (Figura 8) nas mesmas condições dos estudos realizados por SWV sobre o eletrodo de DDB (tampão BR 0,1 mol L-1 e pH =6,0). Foi observada boa linearidade no intervalo de concentração de 0,10 a $3,90 \times 10^{-5} \mathrm{~mol} \mathrm{~L}^{-1}$. Nestas condições o 4-CF apresentou um pico com tempo de retenção em aproximadamente 3,9 $\mathrm{min}$.

Na Tabela 1 encontram-se os valores de LD e os resultados das regressões lineares das curvas analíticas do 4-CF obtidas por SWV e HPLC.

Observa-se na Tabela 1 que o limite de detecção obtido utilizando a cromatografia líquida de alta eficiência é menor do que o obtido utilizando o eletrodo de diamante, no entanto todos os valores encontrados estão dentro do limite máximo de 4-CF permitido em águas pelo Conselho Nacional do Meio Ambiente (CONAMA) ${ }^{29}$ e pelo

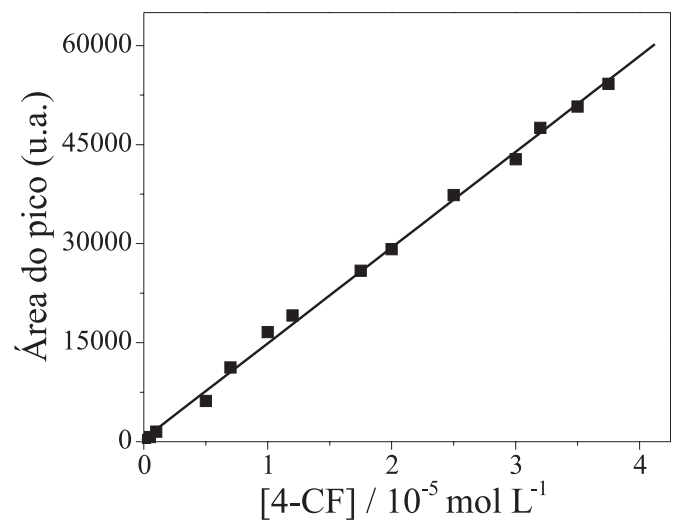

Figura 8. Curva analítica obtida por cromatografia líquida de alta eficiência para o 4-CF em tampão $\mathrm{BR} 0,1 \mathrm{~mol} \mathrm{~L}^{-1}$ e $\mathrm{pH}=6,0$. A área é apresentada em unidades arbitrárias do próprio instrumento 
Tabela 1. Resultados de regressão linear das curvas analíticas e valores de limite de detecção e recuperação obtidos para o 4-CF em água pura, utilizando SWV e HPLC

\begin{tabular}{clc}
\hline Técnica & SWV & HPLC \\
\hline $\mathrm{r}$ & 0,9994 & 0,9998 \\
$\mathrm{~S}_{\mathrm{b}}$ & 0,006 & $0,442^{*}$ \\
$\mathrm{~b}$ & 0,360 & $1,45 \times 10^{9}$ \\
$\mathrm{LD} /\left(\mu \mathrm{g} \mathrm{L}^{-1}\right)$ & 6,40 & 0,01 \\
$\%$ Recuperação & 100,5 & 96,0
\end{tabular}

* O limite de detecção foi calculado utilizando a relação: $L D=3 S_{B} / b$, onde $S_{\mathrm{B}}$ é valor do desvio padrão do intercepto y na curva analítica.

"Environmental Protetion Agency"4 (EPA), que é de $100 \mu \mathrm{g} \mathrm{L}{ }^{-1}$. Desta forma, o eletrodo de diamante dopado com boro e a SWV surgem como uma alternativa à tradicional técnica cromatográfica, com a vantagem das medidas serem realizadas em tempo bem menor e a possibilidade de serem feitas diretamente, sem a necessidade de prétratamentos das amostras.

\section{Efeito de interferentes e experimentos de recuperação aparente}

Para análise do efeito de interferentes foram realizadas medidas com águas coletadas em um poço artesiano e em dois córregos da cidade de São Carlos (ver parte experimental). Para realização das medidas, as amostras de águas foram dopadas com uma certa quantidade de 4-CF e os experimentos de recuperação aparente foram realizados pelo método de adição de padrão.

Na Figura 9 estão apresentadas as curvas analíticas obtidas com o eletrodo de diamante no intervalo de concentração de 0,72 a $3,90 \times 10^{-5} \mathrm{~mol} \mathrm{~L}^{-1}$, em tampão BR $0,1 \mathrm{~mol} \mathrm{~L}^{-1}, \mathrm{pH}=6,0$, usando diferentes amostras de água.

Os resultados de regressão linear obtidos para o 4-CF nas diferentes amostras de água e também os valores de limite de detecção, que foram calculados utilizando-se os mesmos critérios aplicados aos resultados obtidos em água pura, encontram-se na Tabela 2.

Observa-se na Tabela 2 que o aumento de impurezas nas amostras causa um aumento no limite de detecção. Isto ocorre provavelmente em função do aumento do conteúdo de matéria orgânica que pode se adsorver na superfície do eletrodo. Outro fator é a presença de ácidos húmicos dissolvidos na água, que podem interagir com o 4-clorofenol, reduzindo sua concentração na célula eletroquímica. No entanto, para o DDB a interferência não é muito acentuada quan-

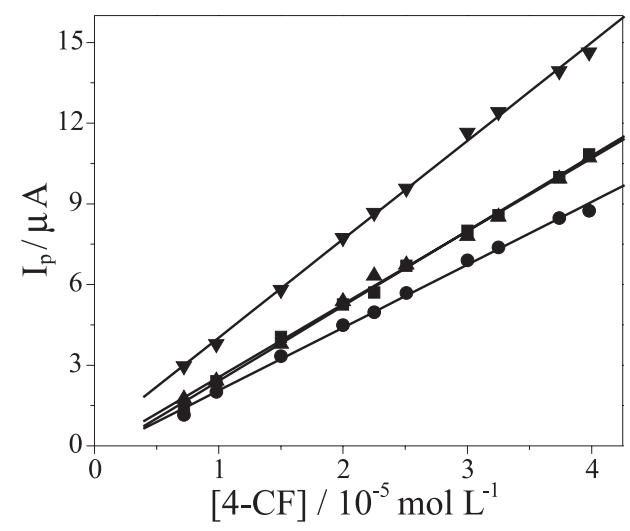

Figura 9. Curvas analíticas obtidas para o 4-CF, nas diferentes amostras de água, $\operatorname{com} f=100 \mathrm{~s}^{-1}, a=50 \mathrm{mV}, \Delta E_{s}=2 \mathrm{mV}$, tampão BR $0,1 \mathrm{~mol} \mathrm{~L}^{-1}$, pH $=$ 6.0. Água pura $(\mathbf{\nabla})$, ponto $1(\mathbf{\Delta})$, ponto $2(\mathbf{\square})$, ponto 3
Tabela 2. Resultados de regressão linear das curvas analíticas do 4clorofenol, obtidos para as águas puras e naturais, utilizando o eletrodo de DDB

\begin{tabular}{lcccc}
\hline Amostra & $\mathrm{r}$ & $\mathrm{S}_{\mathrm{b}} /(\mu \mathrm{A})$ & $\mathrm{b} /\left(\mathrm{A} / \mathrm{mol} \mathrm{L}^{-1}\right)$ & $\mathrm{LD} /\left(\mu \mathrm{g} \mathrm{L}^{-1}\right)$ \\
\hline Milli-Q & 0,9994 & 0,006 & 0,360 & 6,4 \\
Ponto 1 & 0,9983 & 0,009 & 0,279 & 16,2 \\
Ponto 2 & 0,9984 & 0,012 & 0,271 & 17,7 \\
Ponto 3 & 0,9984 & 0,014 & 0,233 & 21,5 \\
\hline
\end{tabular}

do comparada com resultados obtidos com outros materiais eletródicos ${ }^{18}$.

Após a obtenção das curvas analíticas foram realizados os testes de recuperação aparente. Para cada tipo de amostra foram feitas três determinações. Os resultados obtidos referentes aos testes de recuperação encontram-se na Tabela 3 .

Tabela 3. Resultados das curvas de recuperação aparente para o 4$\mathrm{CF}$, obtidas em amostras de águas puras e naturais, utilizando o eletrodo de DDB

\begin{tabular}{lccc}
\hline Amostra & \multicolumn{2}{c}{ 4-CF / $\left(10^{-5} \mathrm{~mol} \mathrm{~L}^{-1}\right)$} & \\
\cline { 2 - 3 } & Adicionado & Encontrado & \% Recuperação \\
\hline Milli-Q & 1,50 & 1,51 & $100,5 \pm 0,6$ \\
Ponto 1 & 1,50 & 1,55 & $103,0 \pm 1,7$ \\
Ponto 2 & 1,50 & 1,52 & $101,3 \pm 1,8$ \\
Ponto 3 & 1,50 & 1,56 & $104,0 \pm 1,4$ \\
\hline
\end{tabular}

Os resultados obtidos indicam que é possível conseguir uma boa porcentagem de recuperação quando se trabalha com o eletrodo de diamante, mesmo em águas naturais.

\section{CONCLUSÕES}

Este trabalho descreve a aplicação com sucesso de uma técnica eletroanalítica para a análise de 4-clorofenol em águas. Também fica evidente que a voltametria de onda quadrada e o eletrodo de diamante dopado com boro podem ser convenientemente utilizados para a determinação do 4-CF em amostras ambientais. Os melhores resultados analíticos com eletrodo de diamante foram obtidos em tampão BR $0,1 \mathrm{~mol} \mathrm{~L}^{-1}, \mathrm{pH}=6,0$, com freqüência da onda quadrada de $100 \mathrm{~s}^{-1}$, amplitude da onda quadrada de $50 \mathrm{mV}$ e incremento de varredura de $2 \mathrm{mV}$. O 4-CF, nestas condições, apresentou somente um pico de oxidação em $0,80 \mathrm{~V}$ vs $\mathrm{Ag} / \mathrm{AgCl}$, com características de processo irreversível e controlado por adsorção das espécies na superfície eletródica. Com os critérios de diagnósticos disponíveis foi possível calcular uma transferência de dois elétrons por molécula de pesticida.

Os limites de detecção obtidos com o eletrodo de diamante dopado com boro foram de $6,4 \mu \mathrm{g} \mathrm{\textrm {L } ^ { - 1 }}$ em água pura e para as águas naturais ficou no intervalo de 16,2 à $21,5 \mu \mathrm{g} \mathrm{L}^{-1}$. Todos estes valores estão dentro do limite máximo de 4-CF permitido em águas pelo Conselho Nacional do Meio Ambiente (CONAMA) e pelo "Environmental Protetion Agency" (EPA) que é de $100 \mu \mathrm{g} \mathrm{L}{ }^{-1}$.

\section{AGRADECIMENTOS}

Os autores agradecem às agências FAPESP, CAPES e CNPq pelas bolsas e apoio financeiro. 


\section{REFERÊNCIAS}

1. Merriman, J. C.; Anthony, D. H. J.; Kraft, J. A.; Wilkinson, R. J.; Chemosphere 1991, 23, 1605.

2. Kristiansen, N.; Froshaug, M.; Aune, K.; Becher, G.; Environ. Sci. Technol. 1994, 28, 1669 .

3. Rogers, K.; Gerlach, C.; Environ. Sci. Technol. 1996, 30, 486A.

4. US. Environmental Protection Agency, Toxic Substance Control Act. 1976.

5. Mussmann, P.; Levsen, K.; Radeck, W.; Fresenius J. Anal. Chem. 1994, 348,654

6. Turnes, M. I.; Mejuto, M. C.; Cela, R.; J. Chromatogr., A 1996, 733, 395

7. Vaz, C. M. P.; Crestana, S.; Machado, S. A. S.; Mazo, L. H.; Massaropi, M. R. C.; Avaca, L. A.; Electroanalysis 1997, 9, 956.

8. Carracedo, M. P. A.; Carra, R. A. G.; Misiego, A. S.; Electroanalysis 2002, $14,433$.

9. Michalkiewicz, S.; Tutaj, M.; Kaczor, M.; Malyszko, J.; Electroanalysis 2002, 14, 297.

10. Zheng, L. Z.; Wu, S. G.; Lin, X. Q.; Nie, L.; Rui, L.; Electroanalysis 2001 13,1351 .

11. Wang, J. L.; Qian, Y.; Chemosphere 1999, 13, 3109.

12. Brillas, E.; Sauleda, R.; Casado, J.; J. Electrochem. Soc. 1998, 145, 759.

13. Azzam, M. O.; Al-Tarazi, M.; Tahboub, Y.; J. Hazard. Mater. 2000, 75, 99.

14. Johnson, S. K.; Houk, L. L.; Feng, J.; Houk, R. S.; Johnson, D.; Environ. Sci. Technol. 1999, 33, 2638.
15. Rodrigo, M. A.; Michaud, P. A.; Duo, I.; Panizza, M.; Cerisola, G.; Comninellis, C.; J. Electrochem. Soc. 2001, 148, D60.

16. Gandini, D.; Michauld, P.; Duo, I.; Maché, E.; Haenni, W.; Perret, A.; Comninellis, C.; New Diamond front. Carbon. Technol. 1999, 5, 9.

17. Rao, T. N.; Fujishima, A.; Diamond Relat. Mater. 2000, 9, 384.

18. Codognoto, L.; Machado, S. A. S.; Avaca, L. A. Em Diamond Mater. VII - The Electrochem. Soc. Proccedings Series; Swain, G. M.; Davidson, J. L.; Angus, J. C.; Ando, T.; Brown, W. D., eds.; Pennigton 2001, 7, 34.

19. Codognoto, L.; Machado, S. A. S.; Avaca, L. A.; Diamond Relat. Mater. 2002, 11, 1670.

20. Rao, T. N.; Loo, B. H.; Sarada, B. V.; Terashima, C.; Fujishima, A.; Anal. Chem. 2002, 74, 1578 .

21. Britton, H. T. S.; Robinson, R. A.; J. Chem. Soc. 1931, 458, 287.

22. Thompson, M.; Ellison, S.; Fajgelj, A.; Wood, R.; Pure Appl. Chem. 1999, 71,337

23. Souza, D.; Machado, S. A. S.; Avaca, L. A.; Quim. Nova 2003, 26, 81

24. Wightman, P. G.; Fein, J. B.; Appl. Geochem. 1999, 14, 319.

25. O`Dea, J. J.; Ribes, A.; Osteryoung, J. G.; J. Electroanal. Chem. 1993, 345, 287.

26. Lovric, M.; Komorsky-Lovric, S.; J. Electroanal. Chem. 1998, 248, 239

27. HyperChem, Molecular Visualization and Simulation; Hypercub: Ontario, 1992.

28. Skoog, D. A.; West, D. M.; Holler, F. L.; Fundamentals of Analytical Chemistry, Saunders College Publishing: USA, 1996.

29. http://www.aga-ambiental.com.br/leis/resoluo\%conama\%2020-86.doc, acessada em Maio 2002 